\title{
Comparación entre trazados cefalométricos manual y digital en radiografías laterales del cráneo
}

\section{Comparison between Manual and Digital Cephalometric Tracing in Side Radiographies of the Skull}

\author{
Janet-Ofelia Guevara-Canales ${ }^{1}$

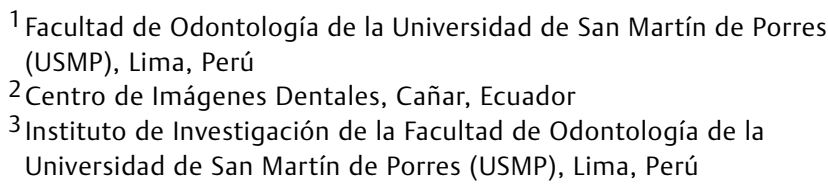

3 Instituto de Investigación de la Facultad de Odontología de la

Eduardo Calle-Velezmoro ${ }^{10}$ Jesica Calle-Morocho ${ }^{2}$ Rafael Morales-Vadillo ${ }^{3}$

Address for correspondence Eduardo Calle-Velezmoro Jr., Andrés Bello, 272, Lima, Perú (e-mail: calle.velezmoro@gmail.com).

Rev Argent Radiol 2021;85:33-40.

\section{Resumen}

Palabras clave

- Cefalometría

- Radiografía Dental Digital

- Radiografía Dental
Objetivo Evaluar la diferencia del trazado cefalométrico manual con el digital en radiografías laterales de cráneo.

Materiales y métodos Inicialmente se realizó un piloto para calibrar al investigador principal, en donde se analizaron 10 radiografías pertenecientes a cinco pacientes del Servicio de Radiología del Centro Odontológico de nuestra institución para evaluar la concordancia entre los dos evaluadores y establecer el tamaño de la muestra. Finalmente, se analizaron 78 radiografías cefalométricas de 39 pacientes, en las que se realizó el trazado cefalométrico de Steiner manual y digital. Esas radiografías se analizaron en una escala 1:1 y posteriormente se evaluó la diferencia de las mediciones mediante el análisis univariado, se determinó en mediana e intervalo intercuartil por separado. En el análisis bivariado, se aplicó la prueba de signo de rangos de Wilcoxon y muestras pareadas para determinar la diferencia de las mediciones cefalométricas de Steiner entre el tipo del análisis cefalométrico. El nivel de significancia se fijó en $\mathrm{p}<0,05$ y confiabilidad al 95\%, respectivamente.

Resultados No hubo diferencia significativa en la mayoría de las mediciones hechas de manera manual y digital, siendo el ángulo interincisal la única medición angular en la cual hubo diferencia significativa al obtener un resultado de $p=0,002$. Mientras tanto, en las mediciones lineales, la única medición en la que hubo diferencia significativa fue en el segmento incisivo inferior - NB con $p=0,005$.

Conclusión No se encuentran diferencias significativas entre el trazado cefalométrico manual y el digital. No obstante, es importante tener en cuenta que la muestra o población evaluada es reducida para definir conclusiones. received

July 5, 2019

accepted

September 8, 2020

published online

May 14, 2021
DOI https://doi.org/

$10.1055 / \mathrm{s}-0040-1721340$

ISSN 1852-9992.

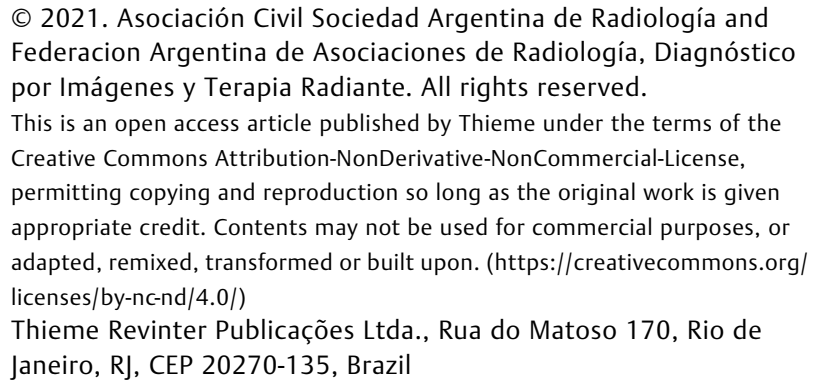

(C) 2021. Asociación Civil Sociedad Argentina de Radiología and Federacion Argentina de Asociaciones de Radiología, Diagnóstico por Imágenes y Terapia Radiante. All rights reserved.

This is an open access article published by Thieme under the terms of the Creative Commons Attribution-NonDerivative-NonCommercial-License, permitting copying and reproduction so long as the original work is given appropriate credit. Contents may not be used for commercial purposes, or adapted, remixed, transformed or built upon. (https://creativecommons.org/ licenses/by-nc-nd/4.0/)

Thieme Revinter Publicações Ltda., Rua do Matoso 170, Rio de Janeiro, RJ, CEP 20270-135, Brazil 


\begin{abstract}
Keywords

- Cephalometry

- Dental Digital Radiography

- Dental Radiography

Objective To evaluate the difference of the manual cephalometric tracing with the digital tracing on lateral cranial radiographs.

Materials and Methods Initially, a pilot study was carried out to calibrate the principal investigator, where 10 radiographs belonging to 5 patients of the Radiology Service of the Dental Center, were analyzed to evaluate the intraclass concordance between the two evaluators. To establish the size of the sample, finally, 78 cephalometric radiographs of 39 patients were analyzed, in which the manual and digital Steiner cephalometric tracing was performed. These radiographs were analyzed on a 1: 1 scale and then the difference of the measurements was evaluated using the univariate analysis, which was determined in the median and interquartile range, separately. In the bivariate analysis, the Wilcoxon rank sign test and paired samples were applied to determine the difference of Steiner's cephalometric measurements between the cephalometric analysis type. The level of significance was set at $\mathrm{p}<0.05$ and $95 \%$ reliability, respectively.

Results There was no significant difference in most of the measurements made manually and digitally. The interincisal angle the only angular measurement in which there was a significant difference when obtaining a result of $p=0.002$, while, in the linear measurements, the only measurement in which there was significant difference was in the lower incisor segment - NB with $\mathrm{p}=0.005$.

Conclusion There is no significant difference between manual and digital cephalometric tracing, nevertheless it is important to keep in mind that the sample or population evaluated is reduced to define conclusions.
\end{abstract}

\section{Introducción}

La cefalometría es una herramienta esencial en el estudio de maloclusiones dentales y discrepancias esqueléticas, cuyo análisis se realiza convencionalmente de forma manual, mediante la identificación de puntos de referencia anatómicos, lo cual es propenso a errores y consume mucho tiempo. ${ }^{1-5}$ Para evitarlo, se han desarrollado programas informáticos capaces de automatizar los análisis cefalométricos. La radiografía digital tiene muchas ventajas respecto de la convencional, entre ellas mejor contraste, nitidez, densidad. Es decir, se obtiene una imagen de mejor calidad que permite, por tanto, una ubicación más exacta de los puntos anatómicos, un diagnóstico adecuado y menor radiación para el paciente. ${ }^{3,6}$

En la actualidad, existen diversas técnicas de análisis cefalométricos, como Ridel o Steiner, ${ }^{7,8}$ entre otros, así como también programas como Nemoceph y Dolphin, entre otros, los cuales se utilizan normalmente para tratamientos ortodóncicos y/o para la elaboración de guías quirúrgicas. ${ }^{2}$ Sin embargo, no se sabe a ciencia cierta si existe diferencia al momento de realizar un trazado cefalométrico digital frente al convencional, teniendo en cuenta las ventajas de la imagen digital frente a una convencional.

Se espera que el desarrollo de esas nuevas tecnologías para la planificación de tratamientos disminuya errores y consuma menos tiempo con una evaluación efectiva y alta reproducibilidad. ${ }^{2}$ Mientras tanto, se siguen usando los trazados convencionales, siendo esa la principal opción en los tratamientos ortodónticos. Mientras no existan estudios que complementen la diferencia entre esas dos técnicas (en las diferentes técnicas de análisis cefalométricos, en los diferentes programas y diferentes poblaciones de estudio), no se podrá implementar de manera definitiva el uso de la cefalometría digital, por lo menos en la población de estudio analizada. Por ello, el objetivo del presente estudio fue evaluar la diferencia del trazado cefalométrico digital con el trazado convencional en radiografías laterales de cráneo.

\section{Materiales y métodos}

El diseño de este estudio fue de tipo observacional, analítico, transversal y retrospectivo, y fue aprobado por el Comité de Ética en Investigación la Facultad de Odontología de nuestra institución.

Inicialmente, se ejecutó un estudio piloto con el objetivo de calibrar al investigador principal en el desarrollo del análisis cefalométrico de Steiner ${ }^{7,8}$ en radiografías digitales y convencionales (estimación de puntos, ángulos y distancias) (-Tabla 1), donde se analizaron 10 radiografías laterales de cinco pacientes que cumplieron con los criterios de inclusión: radiografías de pacientes mayores de 18 años de edad, imágenes de buena calidad y adecuada visualización de estructuras anatómicas relacionadas al área de estudio. Se realizó el análisis cefalométrico de Steiner en una computadora iMac all in one (27" Intel Core i5 8GB 1TB Silver) analizándose cuatro de doce medidas (ángulo GoGn - SN, ángulo interincisal, ANB y distancia Pg - NB), para estimar las concordancias se aplicó el coeficiente de correlación de intraclase. La calibración interobservador se ejecutó entre el investigador principal y un especialista en radiología oral y maxilofacial con 14 años de experiencia y la calibración intraobservador después de cinco 
Tabla 1 Medidas del análisis cefalométrico de Steiner

\begin{tabular}{|l|l|}
\hline MEDIDAS & DEFINICIONES \\
\hline Angulares & $\begin{array}{l}\text { Ángulo formado por los planos } \\
\text { Silla-Nasion (S-N) y Nasion-Punto } \\
\text { A (N-A) }\end{array}$ \\
\hline SNA & $\begin{array}{l}\text { Ángulo formado por los planos } \\
\text { Silla-Nasion (S-N) y Nasion-Punto } \\
\text { B (N-B) }\end{array}$ \\
\hline SNB & $\begin{array}{l}\text { Ángulo formado por los planos } \\
\text { Nasion Punto A (N-A) y Nasion- } \\
\text { Punto B (N-B) }\end{array}$ \\
\hline ANB & $\begin{array}{l}\text { Ángulo formado por los planos } \\
\text { Silla-Nasion (S-N) y Nasion-Punto } \\
\text { D (N-D) }\end{array}$ \\
\hline SND & $\begin{array}{l}\text { Ángulo formado entre el } \\
\text { plano oclusal y el plano S-N }\end{array}$ \\
\hline Ángulo plano \\
oclusal - SN & $\begin{array}{l}\text { Ángulo formado por el plano } \\
\text { mandibular (Go-Gn) y el plano S-N }\end{array}$ \\
\hline Ángulo GoGn - SN & $\begin{array}{l}\text { Ángulo formado por los ejes } \\
\text { longitudinales de los incisivos } \\
\text { superior e inferior }\end{array}$ \\
\hline Ángulo interincisal & $\begin{array}{l}\text { Ángulo formado por el eje } \\
\text { longitudinal del incisivo superior } \\
\text { y el plano N-A }\end{array}$ \\
\hline Áneales & $\begin{array}{l}\text { Ángulo formado por el eje } \\
\text { longitudinal del incisivo inferior } \\
\text { y el plano N-B }\end{array}$ \\
\hline Segmento IS - NA & $\begin{array}{l}\text { Distancia entre el borde incisal } \\
\text { del incisivo superior y el plano N-A }\end{array}$ \\
\hline Segmento II - NB & $\begin{array}{l}\text { Distancia entre el borde incisal } \\
\text { del incisivo inferior y el plano N-B }\end{array}$ \\
\hline Distancia Pg - NB & $\begin{array}{l}\text { Distancia entre el punto } \\
\text { Pg y el plano N-B }\end{array}$ \\
\hline
\end{tabular}

días, dando como resultado: 0,941 y 0,996 respectivamente, es decir, una concordancia casi perfecta.

El estudio piloto permitió además establecer el tamaño mínimo de muestra mediante la fórmula para comparar dos medias apareadas (medidas repetidas) en un solo grupo, obteniendo como tamaño mínimo 30 radiografías (unidades de análisis) por cada tipo de técnica. Finalmente, se decidió tomar un mayor número de tamaño de la muestra, la cual estuvo conformada por 78 radiografías laterales pertenecientes a 39 pacientes (no se incluyeron las radiografías utilizadas en el estudio piloto) que acudieron al Servicio de Radiología del Centro Odontológico de nuestra institución durante el año 2016.

Las radiografías cefalométricas laterales fueron tomadas (como parte del protocolo del registro institucional) por un equipo Pro Max (PlanMeca ${ }^{\circledR}$, Helsinki, Finlandia), con un kilovoltaje de 70 a 76, miliamperaje de 8 a 10 y tiempo de exposición de 8 a 10 segundos; el paciente se ubicó de manera tal que el plano de Frankfurt esté paralelo al piso, se fijan unas varillas del cefalostato en el interior de los oídos para evitar movimientos de la cabeza, se pide al paciente que mantenga la oclusión dental y relaje los labios. Todas las imágenes incluían una regla milimetrada (que luego servirá para la calibración); luego se almacenaron en la base de datos informática Romexis versión 3.6.0, Planmeca, Helsinki, Finlandia, de donde se exportó la imagen en formato JPG.

Para el análisis de Steiner, primero se realizó el análisis cefalométrico digital mediante el software Nemoceph (Nemotec, Madrid, España, 2016, sistema operativo para Windows). Las imágenes digitales fueron analizadas una a una, para lo cual se importa la imagen al programa, se realiza la calibración (digitalizando dos puntos en la regla incluida en la imagen), se selecciona el tipo de análisis (Steiner) y se ubican los puntos anatómicos, dejando que el software genere automáticamente las medidas angulares y lineales (-Fig. 1). Luego se realizó el análisis manual en radiografías impresas exportadas del software con estructuras anatómicas previamente delimitadas, para lo cual se usó una hoja de acetato sobre la radiografía y, con un lápiz, se ubicaron las principales estructuras anatómicas y los puntos anatómicos de referencia con una regla milimetrada se conectan los puntos entre sí y se obtienen líneas y planos, la intersección de ellos genera datos lineales y angulares que fueron medidos utilizando una regla y un graduador (-Fig. 2). Cabe mencionar que los trazados realizados con el software y los trazados manuales (10 pacientes por día) fueron del mismo paciente en una escala de medición de 1:1.

Los datos se registraron utilizando el paquete estadístico Stata versión 14.0 para Windows Corporation (Stata, Texas, EE. UU.). No se cumplió el supuesto de normalidad, (Shapiro-Wilk: $\mathrm{p}<0,05$ ). El análisis univariado de las mediciones cefalométricas de Steiner entre el tipo de radiografía, se determinó en mediana e intervalo intercuartil (IIQ), por separado. En el análisis bivariado, se aplicó la prueba de signo de rangos de Wilcoxon y muestras pareadas para determinar la diferencia de las mediciones cefalométricas de Steiner entre el tipo del análisis cefalométrico. El nivel de significancia se fijó en $\mathrm{p}<0,05$ y confiabilidad al $95 \%$, respectivamente.

\section{Resultados}

Se obtuvieron resultados de mediciones tanto angulares $\left({ }^{\circ}\right)$, lineales $(\mathrm{mm})$, la media con intervalo de confianza al $95 \%$, mediana, desviación estándar, valor mínimo y el valor máximo de cada medición del análisis cefalométrico Steiner en las radiografías laterales trabajadas de manera digital mediante el uso del software Nemoceph, (-Tabla 2), y del trazado manual mediante el uso de radiografías laterales impresas en papel de acetato, (- Tabla 3).

Seguidamente, se compararon ambos trazados cefalométricos para determinar si existía diferencia estadísticamente significativa entre ambas mediciones lineales y angulares. El ángulo interincisal fue la única medición angular en la cual hubo diferencia estadísticamente significativa. Mientras tanto, en las mediciones lineales, la única en la que hubo también esa diferencia fue en el segmento incisivo inferior (Segmento II) - NB (-Tabla 4). Asimismo, cabe mencionar que en esa última medición se obtuvo el valor de concordancia más bajo entre todas las evaluadas con un Coeficiente de Correlación Intraclase $(\mathrm{CCI})=0,809,(-$ Tabla 5 ). 


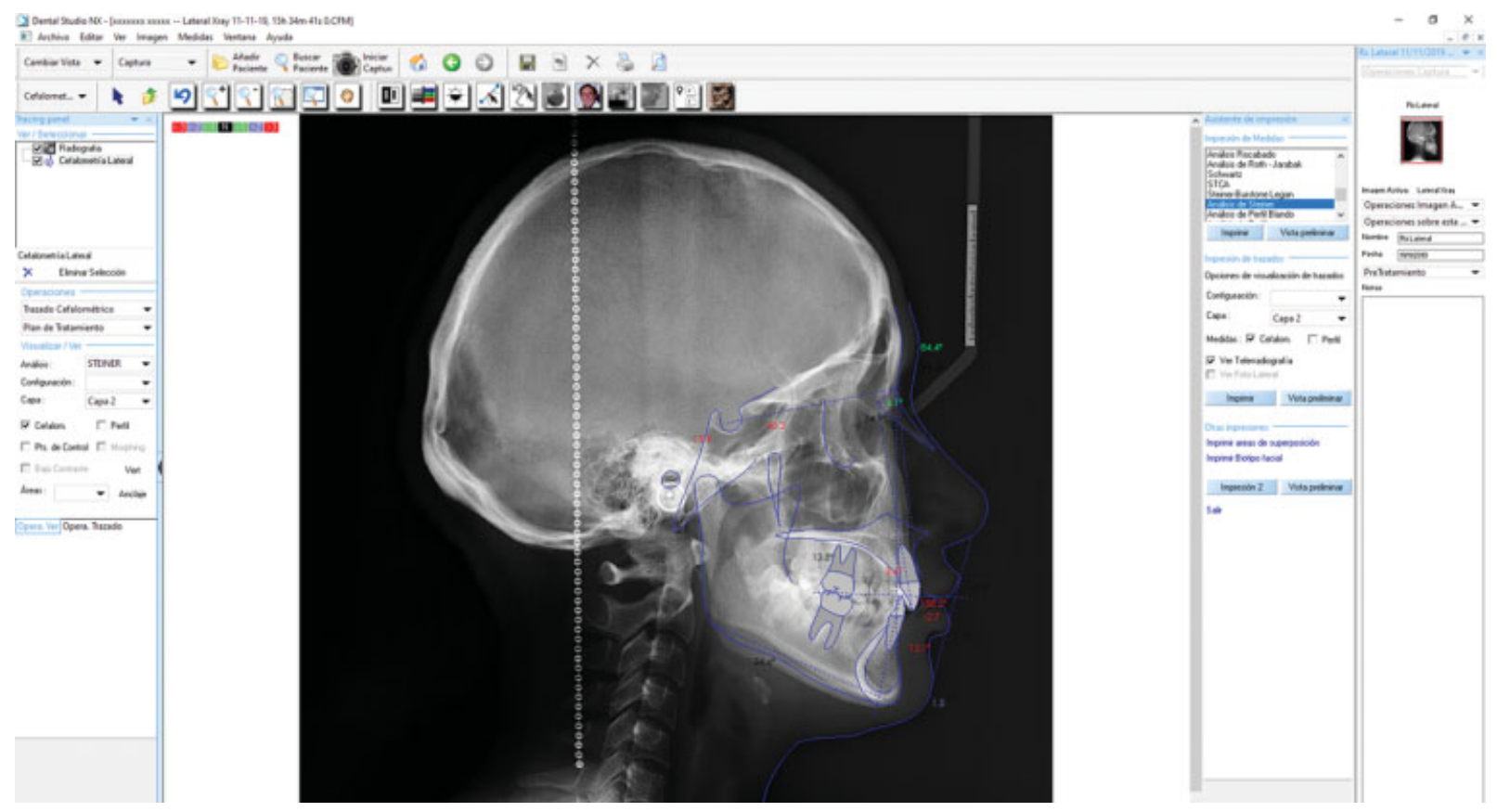

Fig. 1 Análisis cefalométrico digital mediante el uso de Nemoceph. Se evidencia el uso del software Nemoceph para la realización del trazado cefalométricos digital, usando una radiografía lateral en formato JPG.

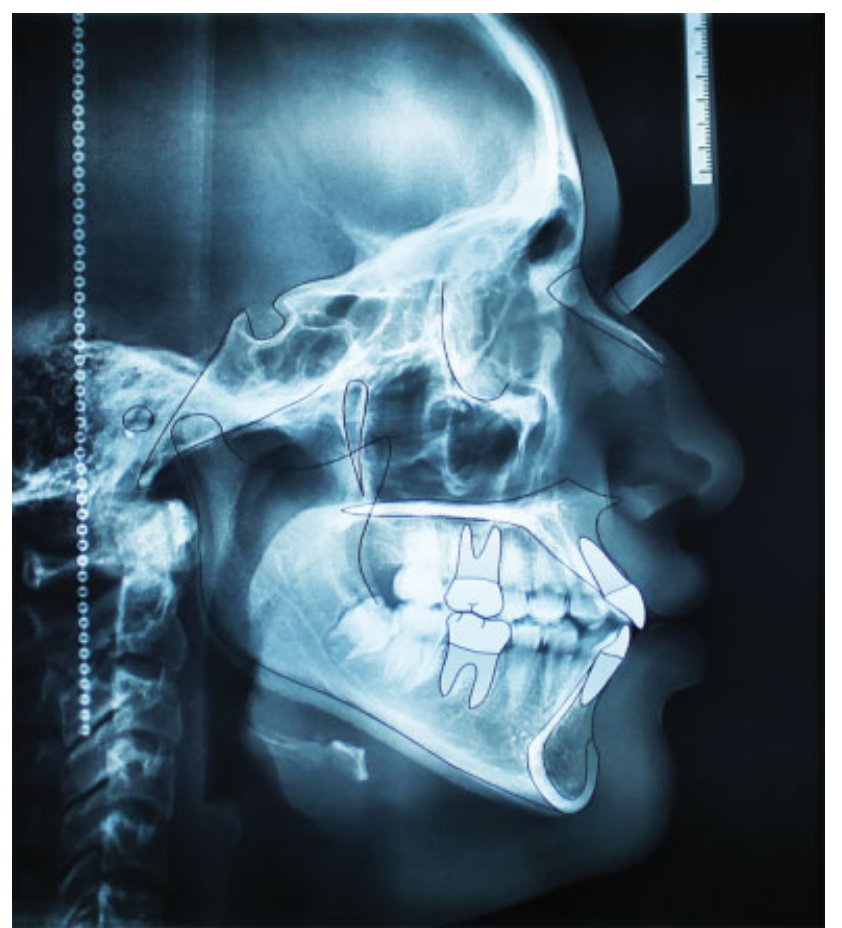

Fig. 2 Análisis cefalométrico manual convencional. Se evidencia el trazado cefalométrico realizado de manera manual, mediante el uso de una radiografía lateral impresa con las estructuras anatómicas principales previamente delimitadas, así como el uso de papel de acetato, regla y transportador.

\section{Discusión}

Determinar la existencia de diferencias significativas entre el trazado cefalométrico manual y digital adquiere importancia con el advenimiento tanto de equipos como softwares digitales que brindan facilidades al momento de realizar los diversos análisis cefalométricos. Además, hay que tener en consideración que, en la era digital, cada día está en aumento el uso de radiografías digitales, imágenes que se deben manipular de la forma más segura posible, lo que se logra mediante el manejo adecuado de los softwares. Ellos tienen varias herramientas, desde aquellas que permiten mejorar una imagen hasta las que permiten realizar un trazado cefalométrico de forma inmediata, ofreciendo probablemente rapidez y eficacia en los diagnósticos, así como también validez y reproducibilidad.

Es por ello que existen diferentes estudios que abarcan el análisis de la diferencia de trazados cefalométricos hechos de manera manual y de manera digital. Sin embargo, dependiendo del autor consultado, varía el tipo análisis cefalométrico (Rickets, Steiner, Tweed, entre otros) y también el tipo de software (Nemoceph, Vistadent, Dolphin, entre otros), aunque existen mediciones angulares y lineales comunes.

Se obtuvieron resultados similares a los de varios autores entre ellos Farooq y col., ${ }^{2}$ cuyas variables mostraron consistencia entre los dos métodos excepto para el eje Y, ángulo incisivo superior (ángulo IS) - NA y ángulo interincisal, esa última medida coincide con el presente estudio.

Esteva y col., ${ }^{6}$ en su estudio de comparación de trazado manual y digital, no encontró diferencias significativas en las medidas SNA, ANB y Pg - NB; al igual que el presente estudio. Sin embargo, Esteva y col., ${ }^{6}$ determinaron que el trazado con el programa digital tiene mayor exactitud en comparación con el método manual.

En El Cairo, AlBarakati y col., ${ }^{9}$ realizaron un estudio similar donde solo se pudo comparar las mediciones angulares SNA, SNB y ANB, siendo esa última la única medición en la cual no se coincidió, debido a que evidenció una diferencia significativa. 
Tabla 2 Análisis descriptivo de las mediciones angulares y lineales de Steiner mediante trazado digital

\begin{tabular}{|c|c|c|c|c|c|c|c|}
\hline \multirow[t]{2}{*}{ MEDICIONES } & \multirow[t]{2}{*}{ Media } & \multicolumn{2}{|c|}{ 95\% I.C. para la media } & \multirow[t]{2}{*}{ Mediana } & \multirow[t]{2}{*}{ D. E. } & \multirow[t]{2}{*}{ Mínimo } & \multirow[t]{2}{*}{ Máximo } \\
\hline & & $\begin{array}{l}\text { Límite } \\
\text { inferior }\end{array}$ & $\begin{array}{l}\text { Límite } \\
\text { superior }\end{array}$ & & & & \\
\hline \multicolumn{8}{|l|}{ Angulares $\left({ }^{\circ}\right)$} \\
\hline SNA & 83.395 & 82.216 & 84.574 & 83.300 & 3.6378 & 75.6 & 91.1 \\
\hline SNB & 79.367 & 77.823 & 80.911 & 80.600 & 4.7631 & 70.1 & 92.8 \\
\hline SND & 76.367 & 74.842 & 77.892 & 76.900 & 4.7044 & 66.9 & 90.0 \\
\hline ANB & 4.067 & 2.929 & 5.205 & 4.800 & 3.5103 & -4.7 & 11.2 \\
\hline Ángulo plano oclusal - SN & 14.931 & 12.818 & 17.044 & 14.800 & 6.5189 & 2.6 & 29.2 \\
\hline Ángulo GoGn - SN & 33.021 & 30.782 & 35.259 & 33.200 & 6.9057 & 14.8 & 48.5 \\
\hline Ángulo incisivo superior - NA & 23.064 & 20.038 & 26.090 & 23.400 & 9.3345 & 0.5 & 39.8 \\
\hline Ángulo incisivo inferior - NB & 28.715 & 25.891 & 31.539 & 30.400 & 8.7118 & 6.3 & 41.1 \\
\hline Ángulo interincisal & 124.338 & 119.476 & 129.201 & 120.100 & 15.0007 & 103.4 & 164.4 \\
\hline \multicolumn{8}{|l|}{ Lineales (mm) } \\
\hline Segmento incisivo superior - NA & 5.300 & 4.207 & 6.393 & 5.500 & 3.3715 & -2.7 & 11.1 \\
\hline Segmento incisivo inferior - NB & 6.595 & 5.513 & 7.677 & 7.100 & 3.3380 & 0.1 & 10.9 \\
\hline Distancia Pogonion - NB & 0.636 & 0.210 & 1.062 & 1.000 & 1.3152 & -4.5 & 3.2 \\
\hline
\end{tabular}

D. E., Desviación Estándar; I.C, Intervalo de Confianza.

Tabla 3 Análisis descriptivo de las mediciones angulares y lineales de Steiner mediante trazado manual

\begin{tabular}{|c|c|c|c|c|c|c|c|}
\hline \multirow[t]{2}{*}{ MEDICIONES } & \multirow[t]{2}{*}{ Media } & \multicolumn{2}{|c|}{ 95\% I.C. para la media } & \multirow[t]{2}{*}{ Mediana } & \multirow[t]{2}{*}{ D. E. } & \multirow[t]{2}{*}{ Mínimo } & \multirow[t]{2}{*}{ Máximo } \\
\hline & & $\begin{array}{l}\text { Límite } \\
\text { inferior }\end{array}$ & $\begin{array}{l}\text { Límite } \\
\text { superior }\end{array}$ & & & & \\
\hline \multicolumn{8}{|l|}{ Angulares $\left({ }^{\circ}\right)$} \\
\hline SNA & 83.410 & 82.288 & 84.532 & 84.000 & 3.4620 & 76.0 & 90.0 \\
\hline SNB & 79.513 & 78.009 & 81.017 & 80.000 & 4.6389 & 71.0 & 91.0 \\
\hline SND & 76.385 & 74.870 & 77.899 & 77.000 & 4.6714 & 67.0 & 90.0 \\
\hline ANB & 4.282 & 3.149 & 5.415 & 5.000 & 3.4940 & -5.0 & 11.0 \\
\hline Ángulo plano oclusal - SN & 14.641 & 12.582 & 16.700 & 15.000 & 6.3515 & 3.0 & 29.0 \\
\hline Ángulo GoGn - SN & 32.051 & 29.872 & 34.231 & 33.000 & 6.7237 & 14.0 & 43.0 \\
\hline Ángulo incisivo superior - NA & 22.897 & 19.906 & 25.889 & 23.000 & 9.2275 & 1.0 & 39.0 \\
\hline Ángulo incisivo inferior - NB & 27.359 & 23.940 & 30.778 & 30.000 & 10.5469 & 3.0 & 41.0 \\
\hline Ángulo interincisal & 123.744 & 118.815 & 128.673 & 120.000 & 15.2051 & 102.0 & 165.0 \\
\hline \multicolumn{8}{|l|}{ Lineales (mm) } \\
\hline Segmento incisivo superior - NA & 5.385 & 4.303 & 6.466 & 6.000 & 3.3373 & -4.0 & 11.0 \\
\hline Segmento incisivo inferior - NB & 7.231 & 5.867 & 8.595 & 8.000 & 4.2081 & 0.0 & 23.0 \\
\hline Distancia Pogonion - NB & 0.641 & 0.258 & 1.024 & 1.000 & 1.1807 & -5.0 & 3.0 \\
\hline
\end{tabular}

D. E., Desviación Estándar; I.C, Intervalo de Confianza.

Otros autores como Murali y col. ${ }^{10}$ no encontraron diferencia significativa en la mayoría de las mediciones hechas de forma manual como digital, al igual que este estudio, solo hubo diferencia significativa en el ángulo interincisal.

En Turquía, Celik y col. ${ }^{11}$ realizaron un estudio utilizando tres métodos, uno manual y dos digitales (uno obtenido directamente y el otro digitalizado previa impresión) en el cual, de todas las mediciones realizadas, solo se encontró diferencia significativa en el ángulo IS - NA y en la medición de incisivo inferior en relación a NB. En el resto de las mediciones no hubo diferencia significativa, hallazgos similares a este estudio, donde las diferencias fueron únicamente para dos medidas (ángulo interincisal y segmento II - NB). Ellos además señalan que la medición cefalométrica computarizada es preferible por sus características de fácil manejo y menor consumo de tiempo.

Tikku y col., ${ }^{3}$ al igual que en este estudio, encontraron diferencia estadísticamente significativa en el Segmento II - 
Tabla 4 Comparación del análisis descriptivo de las mediciones angulares y lineales de Steiner entre el trazado digital y manual

\begin{tabular}{|l|l|l|l|}
\hline MEDICIONES & DIGITAL & CONVENCIONAL & $\begin{array}{l}\text { VALOR } \\
\mathbf{p}\end{array}$ \\
\hline Angulares ( ${ }^{\circ}$ ) & & & \\
\hline SNA & 83.395 & 83.410 & $0.906^{\mathrm{a}}$ \\
\hline SNB & 79.367 & 79.513 & $0.381^{\mathrm{b}}$ \\
\hline SND & 76.367 & 76.385 & $0.899^{\mathrm{a}}$ \\
\hline ANB & 4.067 & 4.282 & $0.063^{\mathrm{a}}$ \\
\hline $\begin{array}{l}\text { Ángulo plano } \\
\text { oclusal - SN }\end{array}$ & 14.931 & 14.641 & $0.315^{\mathrm{a}}$ \\
\hline $\begin{array}{l}\text { Ángulo } \\
\text { GoGn - SN }\end{array}$ & 33.021 & 32.051 & $0.080^{\mathrm{a}}$ \\
\hline $\begin{array}{l}\text { Ángulo incisivo } \\
\text { superior - NA }\end{array}$ & 23.064 & 22.897 & $0.428^{\mathrm{a}}$ \\
\hline $\begin{array}{l}\text { Ángulo incisivo } \\
\text { inferior - NB }\end{array}$ & 28.715 & 27.359 & $0.121^{\mathrm{a}}$ \\
\hline Ángulo interincisal & 124.338 & 123.744 & $0.002^{* \mathrm{~b}}$ \\
\hline Lineales (mm) & & & $0.961^{\mathrm{b}}$ \\
\hline $\begin{array}{l}\text { Segmento incisivo } \\
\text { superior - NA }\end{array}$ & 5.300 & 5.385 & $0.005^{* \mathrm{~b}}$ \\
\hline $\begin{array}{l}\text { Segmento incisivo } \\
\text { inferior - NB }\end{array}$ & 6.595 & 7.231 & 0.641 \\
\hline $\begin{array}{l}\text { Distancia } \\
\text { Pogonion - NB }\end{array}$ & 0.636 & & \\
\hline
\end{tabular}

*Indica significancia estadística $\alpha<0,05$.

${ }^{a} \mathrm{t}$ de Student para muestras relacionadas.

bPrueba de los rangos con signo de Wilcoxon.

$\mathrm{NB}$, pero clínicamente aceptable pues la variación no fue mayor a $1^{\circ}$ para las mediciones angulares y $1 \mathrm{~mm}$ para mediciones lineales.

Todos los estudios previamente mencionados realizaron el análisis cefalométrico manual usando los mismos materiales (negatoscopio y papel de acetato). La diferencia fue en la realización del trazado cefalométrico digital ya que algunos usaron radiografías digitales (directas e indirectas) importadas del software y otros simplemente realizaron el escaneo de radiografías análogas. Eso, junto con el factor humano, podría explicar el motivo por el cual los estudios no coinciden en las mediciones con diferencia significativa entre ambas técnicas.

Otro motivo que hay que tener en cuenta es que las radiografías digitales tienen una infinidad de ventajas, como la manipulación del contraste, nitidez, brillo, zoom, entre otras. También permiten realizar mediciones lineales y angulares en tamaño real, lo cual facilita la ubicación de las estructuras anatómicas para la localización de puntos cefalométricos lo que conlleva a mediciones más exactas y reales.

Concluyendo de la misma manera que Esteva y col., ${ }^{6}$ Celik y col. $^{9}$ y Sayinsu y col., ${ }^{12}$ los cuales mencionan que la cefalometría digital debería ser la primera opción en cuanto al tipo de trazado en odontología, así mismo esa herramienta no solo serviría para la rama de ortodoncia, ya que, como mencionan Lindner y col. ${ }^{13}$ y Sushir y col., ${ }^{14}$ se puede usar para determinar puntos cefalométricos y/o medir
Tabla 5 Coeficientes de correlación intraclase $(\mathrm{CCl})$ con sus intervalos de confianza al $95 \%$ para las mediciones angulares y lineales obtenidas de manera digital y convencional

\begin{tabular}{|l|l|l|l|}
\hline \multirow{2}{*}{ MEDIDA } & \multirow{2}{*}{ CCI } & \multicolumn{2}{|l|}{$\begin{array}{l}\text { INTERVALOS DE } \\
\text { CONFIANZA AL 95\% }\end{array}$} \\
\cline { 3 - 4 } & & $\begin{array}{l}\text { Límite } \\
\text { Inferior }\end{array}$ & $\begin{array}{l}\text { Límite } \\
\text { Superior }\end{array}$ \\
\hline Angulares ( ${ }^{\circ}$ ) & & & \\
\hline SNA & .987 & .975 & .993 \\
\hline SNB & .989 & .979 & .994 \\
\hline SND & .991 & .983 & .995 \\
\hline ANB & .989 & .979 & .994 \\
\hline $\begin{array}{l}\text { Ángulo Plano } \\
\text { Oclusal }\end{array}$ & .981 & .963 & .990 \\
\hline Ángulo Go Gn & .931 & .868 & .964 \\
\hline $\begin{array}{l}\text { Ángulo Incisivo } \\
\text { Superior }\end{array}$ & .995 & .991 & .997 \\
\hline $\begin{array}{l}\text { Ángulo Incisivo } \\
\text { Inferior }\end{array}$ & .915 & .837 & .955 \\
\hline Ángulo Interincisal & .998 & .995 & .999 \\
\hline Lineales (mm) & & & .995 \\
\hline $\begin{array}{l}\text { Segmento Incisivo } \\
\text { Superior }\end{array}$ & .984 & .969 & .991 \\
\hline $\begin{array}{l}\text { Segmento Incisivo } \\
\text { Inferior }\end{array}$ & .809 & .639 & .899 \\
\hline $\begin{array}{l}\text { Distancia } \\
\text { Pogonion }\end{array}$ & .953 & .910 & .975 \\
\hline
\end{tabular}

CCl, Coeficiente de Correlación Intraclase de acuerdo absoluto.

la dimensión vertical. Por tanto, ella es de suma importancia en la realización de todo tratamiento rehabilitador.

Además, hay que tener en consideración los diferentes tipos de software utilizados por parte de otros autores (Radioceph, Dolphin, entre otros) y los diferentes tipos de equipos (Planmeca, Sirona, Morita, entre otros) ya que cada programa $\mathrm{y} / \mathrm{o}$ equipo tienen sus propias herramientas, sistema de funcionamiento y sistema de mediciones.

Recientemente, Calle-Morocho y col. ${ }^{15}$ realizaron un estudio para determinar las discrepancias en las mediciones cefalométricas entre dos tipos de imagen digital (radiografía cefalométrica lateral digital y el cefalograma virtual por Tomografía Computarizada (TC) de Haz Cónico) en 40 pacientes, con la utilización de Nemoceph para los dos tipos de imagen, evaluando la validez y reproducibilidad mediante la comparación por parte del investigador principal del análisis cefalométrico de Steiner, obteniendo diferencias mininas entre el primer y segundo análisis. Eso puede explicarse debido a las ventajas y herramientas con las que cuentan tanto la imagen digital como el trazado cefalométrico digital, los que proporcionan excelente reproducibilidad, tiempo ahorrado (evitando el trazado manual) y eficiencia.

Asimismo, otros estudios han utilizado diferentes tipos de software como Prabhakar y col., ${ }^{4}$ compararon el trazado 
manual con el trazado computarizado con los sistemas Nemoceph y Dolphin sin diferencias estadísticamente significativas entre los métodos computarizados y con diferencias significativas en dos medidas (Co-A y Co-Gn) con el método de trazado manual. Lalakiya y col. $^{5}$ compararon el trazado manual con el digital mediante el uso del software FACAD cuyos resultados mostraron diferencia significativa en seis de dieciocho medidas analizadas, siendo ellas el ángulo facial, ángulo oclusal SN, inclinación del plano oclusal, ángulo Go - Gn a SN, plano $\mathrm{Ar}$ - Go y el ángulo interincisal, siendo ese último un resultado similar a este estudio. Sangroula y col., ${ }^{16}$ evaluaron la confiabilidad y precisión de mediciones cefalométricas lineales y angulares comparando los programas AutoCEPH y Dolphin con el método de rastreo manual, en 17 parámetros cefalométricos. Para ello, evaluaron el CCI con un resultado de 0,813-0,998 en todos los parámetros al comparar los tres métodos. Sin embargo, mencionan que fue mayor el nivel de acuerdo entre las mediciones al usar los softwares computarizados en comparación con los trazados manuales. Bonilla y col., ${ }^{17}$ evaluaron la reproducibilidad y precisión de nueve medidas angulares comparando el trazado manual con el digital mediante el software Cephapoint, analizando el error interobservador entre tres observadores con intervalo de una semana, obteniendo que los promedios de error interobservador entre el trazado manual y el computarizado no presentaron diferencias significativas entre los métodos, con menor diferencia en el ángulo formado por el plano de Frankfort y la línea del punto A al punto Pogonion para el trazado manual y el ángulo de la convexidad de tejidos duros, formado por la intersección de los planos que van del punto Nasion al punto A y del punto A al punto Pogonion para el trazado digital. Aksoy y col., ${ }^{18}$ compararon la confiabilidad de mediciones lineales y angulares por imágenes digitales de radiografías cefalométricas laterales (2D) y por TC de Haz Cónico (3D), usando el software Vista Dent OC (como software 2D) y los softwares In vivo 5.1.2, Maxilim y Romexis (como softwares 3D), obteniendo resultados de $\mathrm{CCI}$ de fiabilidad intraobservador poco confiables con un $\mathrm{p}>0,05$.

Zamora y col., ${ }^{19}$ compararon las mediciones cefalométricas con los programas Nemoceph 3D e In vivo, con resultados sin diferencias estadísticamente significativas entre las mediciones angulares o lineales. Sería ideal que se realizaran más estudios utilizando otros tipos de softwares para comparaciones. En este estudio, así como en los anteriormente mencionados, se pudo determinar que no hay diferencia significativa en la mayoría de las mediciones en relación al trazado cefalométrico de Steiner, más aún teniendo en consideración que este análisis cefalométrico tiene una desviación estándar que varía dependiendo de la medición, siendo en el caso del ángulo interincisal y el Segmento II - NB $\mathrm{de}+/-2^{\circ} / \mathrm{mm}$ respectivamente.

Finalmente, se debe resaltar la importancia y las ventajas que brinda un análisis cefalométrico realizado de manera digital y que, para determinar su implicancia clínica, es necesario que se realicen estudios adicionales, en los cuales se determine si los valores hallados (mediciones angulares y lineales) alteran de una u otra manera el diagnóstico definitivo y si es determinante para la elección del plan de tratamiento. Si bien algunos estudios han indicado que diferencias de hasta $2^{\circ}$ o $2 \mathrm{~mm}$ no representan relevancia clínica, ${ }^{20-22}$ la precisión es importante. Probablemente esos programas digitales proporcionen estimaciones libres de sesgo y más cercanas a la realidad, lo que sería ideal. ${ }^{12}$

\section{Conclusiones}

En el presente trabajo, no encontramos diferencias significativas entre el trazado cefalométrico manual y el digital, aunque es importante tener en cuenta que la muestra o población evaluada es reducida para definir conclusiones.

\section{Responsabilidades éticas}

Protección de personas y animales. Los autores declaran que para esta investigación no se han realizado experimentos en seres humanos ni en animales.

\section{Confidencialidad de los datos}

Los autores declaran que han seguido los protocolos de su centro de trabajo sobre la publicación de datos de pacientes.

Derecho a la privacidad y consentimiento informado Los autores declaran que en este artículo no aparecen datos de pacientes.

Aprobación ética: Informe $\mathrm{N}^{\circ}$ 036-2016-INVE-FO-USMP.

\section{Agradecimientos}

Los autores agradecen al Instituto de Diagnóstico Maxilofacial de Lima, Perú, por brindar las herramientas digitales para el análisis y a la Dra. Karina Pardo por la asesoría.

Conflicto de Intereses

Los autores declaran no tener ningún conflicto de intereses.

\section{Bibliografía}

1 Broadbent BH. A new X-ray technique and its application to orthodontia. Angle Orthod. 1981;51(02):93-114

2 Farooq MU, Khan MA, Imran S, Sameera A, Qureshi A, Ahmed SA, et al. Assessing the Reliability of Digitalized Cephalometric Analysis in Comparison with Manual Cephalometric Analysis. J Clin Diagn Res. 2016;10(10):ZC20-ZC23

3 Tikku T, Khanna R, Maurya RP, Srivastava K, Bhushan R. Comparative evaluation of cephalometric measurements of monitor-displayed images by Nemoceph software and its hard copy by manual tracing. J Oral Biol Craniofac Res. 2014;4(01): 35-41

4 Prabhakar R, Rajakumar P, Karthikeyan MK, Saravanan R, Vikram NR, Reddy A. A hard tissue cephalometric comparative study between hand tracing and computerized tracing. J Pharm Bioallied Sci. 2014;6(05, Suppl 1):S101-S106

5 Lalakiya HBaswaraj, Agrawal C, Patel U, Ramani A, Patel V, Chaudhary S. The Comparison of Computer Aided Digital 
Cephalometric Radiograph with Manual Tracing. Int J Adv Res (Indore). 2016;4(01):621-626

6 Esteva-Segura F, Sanchez-Valverde A, Melendez-Ocampo A, Cedillo-Angelares P. Estudio comparativo entre cefalometría digital y manual con radiografías digitales. Rev Mex Ortodoncia. 2014;2(02):95-98

7 Steiner C. Cephalometrics for you and me. Am J Orthod. 1953;39 (10):729-755

8 Steiner C. Cephalometrics in clinical practice. Angle Orthod. 1959; 29:8-29

9 Albarakati SF, Kula KS, Ghoneima AA. The reliability and reproducibility of cephalometric measurements: a comparison of conventional and digital methods. Dentomaxillofac Radiol. 2012;41(01):11-17

10 Murali R, Sukumai M, Faisal T. Rajalingam. Comparative Study of Manual Cephalometric Tracing in Digital Lateral Cephalogram for Accuracy and Reliability of Landmarks. Indian J Multidiscip Denti. 2011;1(03):126-134

11 Celik E, Polat-Ozsoy O, Toygar Memikoglu TU. Comparison of cephalometric measurements with digital versus conventional cephalometric analysis. Eur J Orthod. 2009;31(03):241-246

12 Sayinsu K, Isik F, Trakyali G, Arun T. An evaluation of the errors in cephalometric measurements on scanned cephalometric images and conventional tracings. Eur J Orthod. 2007;29(01):105-108

13 Lindner C, Wang CW, Huang CT, Li CH, Chang SW, Cootes TF. Fully Automatic System for Accurate Localisation and Analysis of Cephalometric Landmarks in Lateral Cephalograms. Sci Rep. 2016;6:33581

14 Sudhir N, Chittaranjan B, Kumar BA, Taruna M, Kumar MP, Reddy MR. Digital Cephalometric Tracings by PRO-CEPH V3 Software for Comparative Analyses of Vertical Dimension in Edentulous Patients. J Clin Diagn Res. 2015;9(05):ZC01-ZC05
15 Calle-Morocho J, Morales-Vadillo R, Guevara-Canales J, AlvaCuneo C. A comparative study of digital lateral radiography and virtual cone-beam computed assisted cephalogram in cephalometric measurements. J Oral Res. 2018;7(08):308317

16 Sangroula P, Sardana HK, Kharbanda OP, Duggal R. Comparison of reliability and validity of posteroanterior cephalometric measurements obtained from AutoCEPH $\odot$ and dolphin ${ }^{\circledR}$ cephalometric software programs with manual tracing. J Indian Orthod Soc. 2018;52(02):106-114

17 Bonilla MM, Barrera JP, Arroyave ÁP, Díaz ME. Comparación de la reproducibilidad en las mediciones angulares entre trazado manual y computarizado. Rev Fac Odontol Univ Antioq. 2014; 25(02):299-312

18 Aksoy S, Kelahmet U, Hincal E, Oz U, Orhan K. Comparison of linear and angular measurements in CBCT scans using 2D and 3D rendering software. Biotechnol Biotechnol Equip. 2016;30(04): 777-784

19 Zamora N, Llamas JM, Cibrián R, Gandia JL, Paredes V. Cephalometric measurements from 3D reconstructed images compared with conventional 2D images. Angle Orthod. 2011;81 (05):856-864

20 Naoumova J, Lindman R. A comparison of manual traced images and corresponding scanned radiographs digitally traced. Eur J Orthod. 2009;31(03):247-253

21 Chen YJ, Chen SK, Yao JCC, Chang HF. The effects of differences in landmark identification on the cephalometric measurements in traditional versus digitized cephalometry. Angle Orthod. 2004;74 (02):155-161

22 Kumar V, Ludlow J, Soares Cevidanes LH, Mol A. In vivo comparison of conventional and cone beam CT synthesized cephalograms. Angle Orthod. 2008;78(05):873-879 